\title{
OCORRÊNCIA DE COMPLICAÇÕES NO PERÍODO GESTACIONAL EM MULHERES COM IDADE MATERNA AVANÇADA
}

\author{
OCCURRENCE OF COMPLICATIONS \\ IN THE GESTATIONAL PERIOD IN WOMEN \\ OF ADVANCED MATERNAL AGE

\section{APARICIÓN DE COMPLICACIONES EN EL PERÍODO GESTACIONAL EN MUJERES EN EDAD MATERNA AVANZADA}

\author{
Juliane Dias Aldrighi ${ }^{1}$ \\ Suelen da Silva Ribeiro ${ }^{2}$ \\ Andressa Kachel Chemim ${ }^{3}$ \\ Marilene Loewen Wall ${ }^{4}$ \\ Samuel Spiegelberg Zuge ${ }^{5}$ \\ Adriana Aparecida Piler ${ }^{6}$
}

Como citar este artigo: Aldrighi JD, Ribeiro SS, Chemim AK, Wall ML, Zuge SS, Piler AA. Ocorrência de complicações no período gestacional em mulheres com idade materna avançada. Rev baiana enferm. 2021;35:e43083.

Objetivo: analisar a associação entre complicações e idade materna avançada durante a gestação. Método: estudo retrospectivo de abordagem quantitativa baseado na análise de prontuários de mulheres em idade avançada que tiveram parto em um hospital-escola do Sul do Brasil. A coleta ocorreu de 2015 a 2018. Realizaram-se análises recorrendo aos testes Qui-Quadrado de Pearson e/ou exato de Fisher, U de Mann-Whitney e razão de prevalência. Resultados: avaliaram-se 1.336 prontuários. As complicações hipertensão arterial sistêmica pré-gestacional, pré-eclâmpsia e diabetes mellitus gestacional apresentaram maiores médias de idade materna. Mulheres acima de 40 anos apresentaram 1,06 vezes maior probabilidade de desenvolver pré-eclâmpsia e 1,33 vezes de desenvolver crescimento intrauterino restrito. Conclusão: o aumento da idade mostrou relação com complicações, principalmente em gestantes acima de 40 anos.

Descritores: Enfermagem Obstétrica. Idade Materna. Gravidez. Complicações na Gravidez. Gravidez de Alto Risco.

Objective: to analyze the association between complications and advanced maternal age during pregnancy. Method: retrospective study of quantitative approach based on the analysis of medical records of women of advanced age whose delivery occurred in a teaching hospital in southern Brazil. The collection took place from 2015 to 2018.

\footnotetext{
Enfermeira. Universidade Federal do Paraná. Curitiba, Paraná, Brasil. juliane.aldrighi@gmail.com. http://orcid.org/0000-0002-9270-7091.

Enfermeira. Hospital Pilar. Curitiba, Paraná, Brasil. https://orcid.org/0000-000 I-7| I5-7।18.

Enfermeira. Residente do Programa de Residência em Enfermagem Obstétrica da Secretaria de Saúde de Curitiba. Curitiba, Paraná, Brasil. https://orcid.org/00000002-2525-74I4.

4 Enfermeira. Doutora em Enfermagem. Docente da Universidade Federal do Paraná. Curitiba, Paraná, Brasil. https://orcid.org/0000-0003- I839-3896.

5 Enfermeiro. Doutor em Enfermagem. Docente da Universidade Comunitária da Região de Chapecó. Chapecó, Santa Catarina, Brasil. https://orcid.org/0000-0002$0420-9122$.

6 Enfermeira. Mestre em Enfermagem. Enfermeira do Complexo Hospital de Clínicas da Universidade Federal do Paraná. Curitiba, Paraná, Brasil. https://orcid. org/0000-0002-0766-0429.
} 
Analyses were performed using Pearson's Chi-Square and/or Fisher's exact tests, Mann-Whitney U and prevalence ratio. Results: 1,336 medical records were evaluated. Pre-gestational systemic arterial hypertension, preeclampsia and gestational diabetes mellitus presented higher mean maternal age. Women aged over 40 years were 1.06 times more likely to develop preeclampsia and 1.33 times to develop intrauterine growth restriction. Conclusion: the increased age showed a relationship with complications, especially in pregnant women aged over 40 years.

Descriptors: Obstetric Nursing. Maternal Age. Pregnancy. Pregnancy Complications. Pregnancy, High-Risk.

Objetivo: analizar la asociación entre complicaciones y edad materna avanzada durante el embarazo. Método: estudio retrospectivo del enfoque cuantitativo basado en el análisis de registros médicos de mujeres en edad avanzada que tuvieron parto en un hospital docente en el sur de Brasil. La colección tuvo lugar de 2015 a 2018. Los análisis se realizaron utilizando chi-square de Pearson y/o las pruebas exactas de Fisher, Mann-Whitney U y la relación de prevalencia. Resultados: se evaluaron 1.336 registros médicos. La hipertensión arterial sistémica pre gestacional, la preclamsia y la diabetes mellitus gestacional presentaron una edad materna media más alta. Las mujeres mayores de 40 años tenían 1,06 veces más probabilidades de desarrollar preclamsia y 1,33 veces de desarrollar crecimiento intrauterino restringido. Conclusión: el aumento de la edad mostró una relación con complicaciones, especialmente en mujeres embarazadas mayores de 40 años de edad.

Descriptores: Enfermeria Obstétrica. Edad Materna. Embarazo. Complicaciones del Embarazo. Embarazo de Alto Riesgo.

\section{Introdução}

A gestação tardia ou gestação em idade materna avançada, que ocorre em mulheres com idade igual ou superior a 35 anos, está relacionada a complicações no período gestacional devido à maior probabilidade de evoluir com desfechos negativos. Assim, é considerada pelo Ministério da Saúde (MS) como gestação de alto risco $^{(1)}$. As complicações decorrem tanto da própria senescência ovariana quanto da frequência aumentada de doenças crônicas devido à idade. Dessa forma, à medida que a gestação é adiada, maior é a suscetibilidade da mulher a riscos nos períodos gestacional e puerperal ${ }^{(2-3)}$.

Nesse sentido, estudos apontam, em relação à mãe, risco aumentado para síndromes hipertensivas - entre elas pré-eclâmpsia (PE) e eclâmpsia, diabetes mellitus gestacional (DMG), hemorragias, óbito fetal (OF). Quanto aos bebês, o risco aumenta para baixo peso ao nascer, prematuridade e baixo índice de APGAR ${ }^{(2-4)}$.

De acordo com a literatura, nas últimas décadas, a incidência de gestações nessa faixa etária vem aumentando, entre outros motivos, devido à criação de políticas voltadas à saúde da mulher e ao incentivo ao planejamento reprodutivo. Isso possibilitou às mulheres a conquista de maior espaço no mercado de trabalho e melhor nível educacional. Assim, passaram a postergar a gestação, a fim de alcançar objetivos na carreira profissional $^{(5)}$.

No Brasil, com o passar dos anos, o número de filhos está cada vez menor, assim como as taxas de natalidade e fecundidade. A despeito disso, observa-se aumento no número de nascidos vivos provenientes de mulheres com 35 anos ou mais. Conforme pesquisa realizada no banco de dados do Departamento de Informática do Sistema Único de Saúde (DATASUS), em 2007, do total de nascidos vivos no país, cerca de $9,7 \%$ eram de gestantes em idade materna avançada. Ao comparar esse dado com o ano de 2017, o número de nascimentos aumentou para $14,4 \%$, o que configura aumento de mais de 50\%. Assim, ao considerar que a média de nascimentos geral não sofreu alteração, tem-se uma tendência de aumento progressivo de nascimentos nessa população ${ }^{(6)}$.

Diante disso, entende-se que o enfermeiro tem papel determinante e central no percurso gestacional dessas mulheres, pois realiza o direcionamento dos cuidados de enfermagem na atenção pré-natal, parto e puerpério, além de atuar em ações de educação em saúde que visam à prevenção e identificação precoce de agravos 
à saúde das gestantes ${ }^{(7)}$. É escassa a produção científica na área da enfermagem sobre a gestação em idade avançada e suas consequências.

Tendo em vista o aumento dessa população em âmbito mundial nos últimos anos e a tendência à manutenção do adiamento das gestações para idades mais avançadas, considera-se que esta temática não se encontra esgotada. Portanto, justifica-se este estudo, devido ao cunho emergente do tema para a Enfermagem e aos poucos subsídios para o cuidado de enfermagem voltado a essa população. Assim, o objetivo deste estudo é analisar a associação entre complicações e idade materna avançada durante a gestação.

\section{Método}

Trata-se de um estudo retrospectivo de abordagem quantitativa, norteado pela ferramenta STROBE (Strengthening the Reporting of $\mathrm{Ob}$ servational Studies in Epidemiology - Fortalecimento do Relato de Estudos Observacionais em Epidemiologia). Esta pesquisa tem base em registro de prontuário e foi desenvolvida em um hospital federal de ensino da Região Sul do Brasil, de atenção terciária, público, cogerido pela Empresa Brasileira de Serviços Hospitalares (Ebserh) desde 2014, referência no atendimento ambulatorial e hospitalar à gestação de alto risco.

Foram incluídos no estudo prontuários de mulheres com 35 anos ou mais, que tiveram parto na instituição, entre os anos de 2011 e 2017. Dentro do universo de 1.796 partos de mulheres com idade igual ou maior de 35 anos nesse período, foi avaliada uma amostra 1.336 mulheres (erro amostral de 2,5\% e nível de significância de 95\%). Os prontuários foram selecionados por amostragem aleatória estratificada.

A coleta de dados foi realizada de dezembro de 2015 a outubro de 2018, de modo informatizado por meio de questionário estruturado, construído no software Epi Info 7 e operacionalizada por duas alunas, uma bolsista e outra voluntária, ambas do Programa de Iniciação Científica do Conselho Nacional de Desenvolvimento Científico e Tecnológico (CNPq). As alunas tiveram uma capacitação que consistiu em apresentação do projeto de pesquisa e explicação quanto ao uso da ferramenta para a coleta. Houve reunião para simulação da coleta dos dados, com o formulário inserido no software, bem como momentos para dirimir dúvidas. Após essas etapas, foram coletados em campo 10 testes pilotos, para avaliar a funcionalidade do formulário no software. Todos os prontuários-teste foram incluídos na amostra.

O questionário de pesquisa com 25 perguntas foi elaborado com base na ficha do Sistema de Acompanhamento do Programa de Humanização no Pré-Natal e Nascimento (SISPRENATAL), abrangendo variáveis sociodemográficas, obstétricas e clínicas, as quais dividiram-se em:

a) sociodemográficas: idade materna avançada (35 a 40 anos; > 40 anos); estado civil (convive com o companheiro ou esposo; separada, divorciada ou viúva; solteira); cor/raça (branca; preta; amarela; parda; ignorada); escolaridade (analfabeta; < 8 anos; 8-12 anos; > 12 anos; ignorada); renda ( $\leq$ um salário mínimo; dois a três salários mínimos; > três salários mínimos; ignorada);

b) obstétricas: idade gestacional (em que foram considerados recém-nascidos pré-termo, os com menos de 37 semanas; a termo, de 37 a 41 semanas incompletas; e pós-termo, os com 42 semanas ou mais); número de consultas durante o pré-natal (menos de seis consultas; seis consultas ou mais); paridade (primípara - sem partos anteriores; multípara - com, pelo menos, um parto anterior); tipo de parto (vaginal, cesárea);

c) clínicas: complicação na gestação atual (PE; eclâmpsia; DMG; trabalho de parto prematuro; placenta prévia (PP); hemorragias; crescimento intrauterino restrito (CIUR); OF; obesidade); complicações pré-existentes (hipertensão arterial sistêmica (HAS); diabetes mellitus (DM); tireoidopatias; cardiopatias; vírus da imunodeficiência humana (HIV); doenças respiratórias; transtornos psiquiátricos; sífilis; CIUR em gestação anterior). 
Justifica-se a utilização dessas complicações por serem as mais citadas como de maior risco, quando considerada a faixa etária de 35 anos ou mais ${ }^{(2,4)}$.

A análise foi realizada no software IBM SPSS versão 18.0 for Windows com análises de frequência (distribuição absoluta e percentual). A fim de avaliar a relação entre a idade materna, os dados sociodemográficos e as complicações no período gestacional, recorreu-se a análises bivariadas, por meio do teste Qui-Quadrado de Pearson $\left(x^{2}\right)$. Nos casos em que os requisitos para aplicação do $x^{2}$ não foram atendidos, aplicou-se o teste exato de Fisher.

Além disso, fez-se a avaliação da normalidade da variável idade materna avançada, por meio do teste de Komolgorov-Smirnov, o qual não atendeu ao requisito da normalidade dos dados ( $p$-valor $<0,000$ ). Após, foram realizadas comparações de média da idade materna avançada e as complicações maternas por meio do teste $\mathrm{U}$ de Mann-Whitney. Para avaliar a associação entre a idade materna avançada e as complicações durante a gestação, aplicou-se o teste de razão de prevalência. O nível de significância assumido nos testes foi de $5 \%$ (p-valor $<0,05$ ).

A pesquisa faz parte de um projeto maior aprovado pelo Comitê de Ética da instituição pesquisada, sob os Pareceres n.o 1.155.166/2015 e n. 3.182.968/2019, e Certificado de Apresentação de Apreciação Ética n. ${ }^{\circ} 46154615.7 .0000 .0096$. Não houve assinatura de Termo de Consentimento Livre Esclarecido por tratar-se de pesquisa em prontuários. Contudo, os demais aspectos da Resolução n.․․ 466/2012, do Conselho Nacional de Saúde, foram respeitados na íntegra.

\section{Resultados}

Foram avaliados prontuários de 1.336 mulheres com idade materna avançada no período de 2011 a 2017. A média da idade foi de 39,5 anos $( \pm 3,06)$, com mínima e máxima de 35 e 51 anos, respectivamente. Houve predomínio da faixa etária de 35 a 40 anos, com 889 mulheres $(66,5 \%)$. Em relação às características sociodemográficas e obstétricas, apenas a escolaridade mostrou relação com a idade materna avançada $(\mathrm{p}=0,011)($ Tabela 1$)$.

Tabela 1 - Características sociodemográficas e obstétricas de mulheres em idade materna avançada. Curitiba, Paraná, Brasil - 2015-2018. (N=1.336)

(continua)

\begin{tabular}{|c|c|c|c|c|c|}
\hline \multirow{2}{*}{ Variável } & \multirow{2}{*}{$\mathbf{n}$} & \multirow{2}{*}{$\%$} & \multicolumn{2}{|c|}{ Idade Avançada } & \multirow{2}{*}{$\mathbf{p}^{*}$} \\
\hline & & & 35 a 40 anos & $>40$ anos & \\
\hline \multicolumn{5}{|l|}{$\operatorname{Raça} / \operatorname{cor}(n=1.320)$} & \multirow[t]{4}{*}{0,145} \\
\hline Branca & 1.161 & 88 & 765 & 396 & \\
\hline Preta & 113 & 8,6 & 83 & 30 & \\
\hline Amarela ou Parda & 46 & 3,4 & 27 & 19 & \\
\hline \multicolumn{5}{|l|}{ Estado Civil (n=1.328) } & \multirow[t]{4}{*}{0,073} \\
\hline Convive com o companheiro ou esposo & 1.029 & 77,5 & 684 & 345 & \\
\hline Separada, divorciada ou viúva & 78 & 5,9 & 44 & 34 & \\
\hline Solteira & 221 & 16,6 & 156 & 65 & \\
\hline \multicolumn{5}{|l|}{ Escolaridade $(n=1.196)$} & \multirow[t]{5}{*}{0,011} \\
\hline Analfabeta & 18 & 1,5 & 11 & 7 & \\
\hline$<8$ anos & 253 & 21,2 & 148 & 105 & \\
\hline 8 a 12 anos & 750 & 62,7 & 497 & 253 & \\
\hline$>12$ anos & 175 & 14,6 & 129 & 46 & \\
\hline \multicolumn{5}{|l|}{ Renda $(n=415)$} & \multirow[t]{4}{*}{0,547} \\
\hline Menor ou igual a 1 salário mínimo & 67 & 16,1 & 39 & 28 & \\
\hline De 2 a 3 salários mínimos & 222 & 53,5 & 145 & 77 & \\
\hline Mais de 3 salários mínimos & 126 & 30,4 & 82 & 44 & \\
\hline \multicolumn{6}{|l|}{ Paridade } \\
\hline Primípara & 244 & 18,3 & 170 & 74 & \multirow[t]{2}{*}{0,241} \\
\hline Multipara & 1.092 & 81,7 & 718 & 374 & \\
\hline
\end{tabular}


Tabela 1 - Características sociodemográficas e obstétricas de mulheres em idade materna avançada. Curitiba, Paraná, Brasil - 2015-2018. (N=1.336)

\begin{tabular}{|c|c|c|c|c|c|}
\hline \multirow{2}{*}{ Variável } & \multirow{2}{*}{$\mathbf{n}$} & \multirow{2}{*}{$\%$} & \multicolumn{2}{|c|}{ Idade Avançada } & \multirow{2}{*}{$\mathbf{p}^{*}$} \\
\hline & & & 35 a 40 anos & $>40$ anos & \\
\hline Tipo de Parto & & & & & 0,107 \\
\hline Vaginal & 556 & 41,6 & 383 & 173 & \\
\hline Cesárea & 780 & 58,4 & 505 & 275 & \\
\hline Idade Gestacional do parto $(n=1.325)$ & & & & & 0,862 \\
\hline Pré-termo & 284 & 21,4 & 185 & 99 & \\
\hline Termo & 1.005 & 75,8 & 672 & 333 & \\
\hline Pós-termo & 36 & 2,7 & 24 & 12 & \\
\hline
\end{tabular}

Fonte: Elaboração própria.

*Teste Qui-Quadrado de Pearson

Em relação à avaliação das doenças pré-gestacionais, foi possível identificar que 728 (54,5\%) apresentaram algum tipo. Na comparação entre a média de idade e as doenças pré-gestacionais, as mulheres que apresentavam HAS antes da gestação tiveram maiores médias de idade $(\mathrm{p}=0,013)($ Tabela 2).

Tabela 2 - Comparação de médias entre as doenças pré-gestacionais e a idade materna avançada. Curitiba, Paraná, Brasil - 2015-2018. (N=1.336)

\begin{tabular}{|c|c|c|c|c|c|}
\hline \multirow{2}{*}{ Doenças pré-gestacionais } & \multicolumn{5}{|c|}{ Idade materna avançada } \\
\hline & $\mathbf{n}$ & $\%$ & Média & Desvio Padrão & $\mathbf{p}^{*}$ \\
\hline \multicolumn{6}{|l|}{ Hipertensão Arterial Sistêmica } \\
\hline Não & 1.036 & 77,5 & 39,40 & 3,05 & 0,013 \\
\hline $\operatorname{Sim}$ & 300 & 22,5 & 39,86 & 3,06 & \\
\hline Diabetes mellitus & & & & & 0,971 \\
\hline Não & 1.250 & 93,6 & 39,51 & 3,06 & \\
\hline $\operatorname{Sim}$ & 86 & 6,4 & 37,33 & 3,02 & \\
\hline Tireoideopatias & & & & & 0,801 \\
\hline Não & 1.125 & 84,2 & 39,48 & 3,01 & \\
\hline $\operatorname{Sim}$ & 211 & 15,8 & 39,64 & 3,29 & \\
\hline Cardiopatias & & & & & 0,145 \\
\hline Não & 1.270 & 84,2 & 39,53 & 3,07 & \\
\hline Sim & 66 & 15,8 & 38,94 & 2,76 & \\
\hline HIV & & & & & 0,823 \\
\hline Não & 1.277 & 95,6 & 39,50 & 3,03 & \\
\hline Sim & 59 & 4,4 & 39,58 & 3,62 & \\
\hline Doenças Respiratórias & & & & & 0,418 \\
\hline Não & 1.286 & 96,3 & 39,52 & 3,07 & \\
\hline $\operatorname{Sim}$ & 50 & 3,7 & 39,02 & 2,96 & \\
\hline Transtornos Psiquiátricos & & & & & 0,060 \\
\hline Não & 1.140 & 85,3 & 39,45 & 3,07 & \\
\hline Sim & 196 & 14,7 & 39,82 & 2,96 & \\
\hline Sifilis & & & & & 0,437 \\
\hline Não & 1.312 & 98,2 & 39,50 & 3,06 & \\
\hline $\operatorname{Sim}$ & 24 & 1,8 & 40,00 & 3,30 & \\
\hline Crescimento intrauterino restrito & & & & & 0,078 \\
\hline$(n=949)$ & 924 & 97,4 & 39,27 & 3,07 & \\
\hline Não & 25 & 2,6 & 40,08 & 2,50 & \\
\hline Sim & & & & & \\
\hline
\end{tabular}

Fonte: Elaboração própria.

*Teste U de Mann-Whitney. 
Ao comparar as médias de idade materna com as complicações durante a gestação foi possível identificar que, estatisticamente, as mulheres que manifestaram PE $(p=0,017)$ e DMG $(p=0,026)$ apresentavam maiores médias de idade. As demais variáveis não apresentaram diferenças estatísticas (Tabela 3).

Tabela 3 - Comparação de médias entre as complicações maternas durante a gestação e a idade materna avançada. Curitiba, Paraná, Brasil - 2015-2018. (N=1.336)

\begin{tabular}{|c|c|c|c|}
\hline \multirow{2}{*}{$\begin{array}{l}\text { Complicação materna durante a } \\
\text { gestação }\end{array}$} & \multicolumn{3}{|c|}{ Idade materna avançada } \\
\hline & Média & Desvio Padrão & $\mathbf{p}^{*}$ \\
\hline Pré-eclâmpsia & & & 0,017 \\
\hline Não & 39,39 & 2,98 & \\
\hline Sim & 39,98 & 3,34 & \\
\hline Eclâmpsia & & & 0,063 \\
\hline Não & 39,51 & 3,06 & \\
\hline $\operatorname{Sim}$ & 37,33 & 2,73 & \\
\hline Diabetes mellitus gestacional & & & 0,026 \\
\hline Não & 39,40 & 3,05 & \\
\hline $\operatorname{Sim}$ & 39,82 & 3,07 & \\
\hline Trabalho de parto prematuro & & & 0,490 \\
\hline Não & 39,48 & 3,03 & \\
\hline Sim & 39,92 & 3,48 & \\
\hline Placenta Prévia & & & 0,230 \\
\hline Não & 39,50 & 3,07 & \\
\hline Sim & 39,90 & 2,37 & \\
\hline Hemorragias & & & 0,657 \\
\hline Não & 39,50 & 3,62 & \\
\hline Sim & 39,65 & 2,94 & \\
\hline Óbito Fetal & & & 0,853 \\
\hline Não & 39,51 & 3,07 & \\
\hline Sim & 39,47 & 2,93 & \\
\hline Oligo/polidrâmnio & & & 0,537 \\
\hline Não & 39,49 & 3,06 & \\
\hline Sim & 39,65 & 3,00 & \\
\hline Obesidade $(n=653)$ & & & 0,421 \\
\hline Não & 38,86 & 2,79 & \\
\hline Sim & 38,33 & 2,17 & \\
\hline
\end{tabular}

Fonte: Elaboração própria.

*Teste U de Mann-Whitney.

Ao avaliar a relação entre as complicações e a idade materna, identificou-se que a idade materna avançada apresentou relação estatística significativa somente com a PE (p=0,009). Já ao avaliar a razão de prevalência entre as complicações e a idade materna, foi possível identificar que as mulheres com mais de 40 anos tinham 1,33 vezes mais probabilidade de desenvolver CIUR (1,33; IC95\%: 1,31 - 1,36) do que aquelas com idade entre 35 e 40 anos (Tabela 4).

Tabela 4 - Associação entre complicações maternas durante a gestação e idade materna avançada. Curitiba, Paraná, Brasil - 2015-2018. (N=1.336)

\begin{tabular}{|c|c|c|c|c|c|c|}
\hline \multirow{3}{*}{$\begin{array}{l}\text { Complicação materna durante } \\
\text { a gestação }\end{array}$} & \multicolumn{4}{|c|}{ Idade Materna Avançada } & \multirow{3}{*}{$\mathbf{p}^{*}$} & \multirow{3}{*}{$\begin{array}{c}\text { Razão de Prevalência } \\
\text { (Intervalo de } \\
\text { Confiança a 95\%) }\end{array}$} \\
\hline & \multicolumn{2}{|c|}{35 a 40 anos } & \multicolumn{2}{|c|}{$>40$ anos } & & \\
\hline & $\mathbf{n}$ & $\%$ & $\mathbf{n}$ & $\%$ & & \\
\hline \multicolumn{5}{|l|}{ Pré-eclâmpsia } & \multicolumn{2}{|l|}{0,009} \\
\hline Não & 731 & 68,1 & 342 & 31,9 & & 1 \\
\hline $\operatorname{Sim}$ & 157 & 59,7 & 106 & 40,3 & & $1,06(1,02-1,12)$ \\
\hline
\end{tabular}


Tabela 4 - Associação entre complicações maternas durante a gestação e idade materna avançada. Curitiba, Paraná, Brasil - 2015-2018. (N=1.336)

(conclusão)

\begin{tabular}{|c|c|c|c|c|c|c|}
\hline \multirow{3}{*}{$\begin{array}{l}\text { Complicação materna durante } \\
\text { a gestação }\end{array}$} & \multicolumn{4}{|c|}{ Idade Materna Avançada } & \multirow{3}{*}{$\mathbf{p}^{*}$} & \multirow{3}{*}{$\begin{array}{c}\text { Razão de Prevalência } \\
\text { (Intervalo de } \\
\text { Confiança a 95\%) }\end{array}$} \\
\hline & \multicolumn{2}{|c|}{35 a 40 anos } & \multicolumn{2}{|c|}{$>40$ anos } & & \\
\hline & $\mathbf{n}$ & $\%$ & $\mathbf{n}$ & $\%$ & & \\
\hline \multicolumn{5}{|l|}{ Eclâmpsia } & \multicolumn{2}{|l|}{$0,448^{\dagger}$} \\
\hline Não & 883 & 66,4 & 447 & 33,6 & & 1 \\
\hline $\operatorname{Sim}$ & 5 & 83,3 & 1 & 16,7 & & $0,87(0,68-1,13)$ \\
\hline \multicolumn{5}{|l|}{ Diabetes mellitus gestacional } & 0,059 & \\
\hline Não & 688 & 67,6 & 329 & 32,4 & & 1 \\
\hline Sim & 200 & 62,7 & 119 & 37,3 & & $1,04(0,99-1,08)$ \\
\hline \multicolumn{7}{|l|}{ Trabalho de parto prematuro } \\
\hline Não & 839 & 66,5 & 422 & 33,5 & 0,460 & 1 \\
\hline $\operatorname{Sim}$ & 49 & 65,3 & 26 & 34,7 & & $1,01(0,93-1,10)$ \\
\hline Placenta Prévia & & & & & 0,089 & \\
\hline Não & 873 & 66,8 & 434 & 33,2 & & 1 \\
\hline $\operatorname{Sim}$ & 15 & 51,7 & 14 & 48,3 & & $1,11(0,98-1,26)$ \\
\hline Hemorragias & & & & & 0,116 & \\
\hline Não & 878 & 66,7 & 438 & 33,3 & & 1 \\
\hline Sim & 10 & 50,0 & 10 & 50,0 & & $1,12(0,97-1,30)$ \\
\hline \multicolumn{5}{|l|}{ Crescimento intrauterino } & 0,308 & \\
\hline restrito & 875 & 66,6 & 438 & 33,4 & & 1 \\
\hline Não & 13 & 56,5 & 10 & 43,5 & & $1,33(1,31-1,36)$ \\
\hline Sim & & & & & & \\
\hline \multicolumn{5}{|l|}{ Óbito Fetal } & 0,799 & \\
\hline Não & 851 & 66,5 & 428 & 33,5 & & 1 \\
\hline $\operatorname{Sim}$ & 37 & 64,9 & 20 & 35,1 & & $1,01(0,92-1,11)$ \\
\hline \multicolumn{5}{|l|}{ Oligo/polidrâmnio } & 0,969 & \\
\hline Não & 834 & 66,5 & 421 & 33,5 & & 1 \\
\hline Sim & 54 & 66,7 & 27 & 33,3 & & $1,00(0,92-1,08)$ \\
\hline \multicolumn{5}{|l|}{ Obesidade $(n=653)$} & 0,154 & \\
\hline Não & 456 & 75,0 & 152 & 25,0 & & 1 \\
\hline Sim & 38 & 84,4 & 7 & 15,6 & & $0,92(0,84-1,02)$ \\
\hline
\end{tabular}

Fonte: Elaboração própria.

*Teste Qui-Quadrado de Pearson. ${ }^{\dagger}$ Teste exato de Fisher.

\section{Discussão}

As complicações HAS pré-gestacional, PE e DMG tiveram relação com maiores médias de idade materna. Além disso, mulheres com idade acima de 40 anos apresentaram maior probabilidade de desenvolver PE e CIUR.

As literaturas brasileira e internacional trazem as síndromes hipertensivas na gestação (hipertensão gestacional, PE, eclâmpsia, síndrome de HELLP) e DMG como complicações predominantes em gestantes de idade avançada. A HAS é o principal fator de risco para esse tipo de intercorrência $^{(2,4,8-10)}$, assim como CIUR é um desfecho comum associado a desordens placentárias causadas por problemas circulatórios/vasculares ${ }^{(11-13)}$.
Nesse sentido, as complicações aqui encontradas formam um "emaranhado" fisiopatológico de causas e consequências simultâneas.

Quanto ao CIUR, apresentou 1,33 vezes probabilidade de ocorrer em mulheres com mais de 40 anos. Essa complicação é causada por má perfusão placentária relacionada a problemas vasculares. Das causas maternas, as doenças hipertensivas são os fatores que mais se associam a casos graves de CIUR, pois afetam a transferência de nutrientes e oxigênio para o feto. As condições clínicas maternas que mais causam CIUR são PE, DM e HAS ${ }^{(1,14)}$. Nessa direção, uma revisão sistemática ${ }^{(2)}$ encontrou a taxa de CIUR de $4 \%$ para 74 estudos analisados. Apesar da pequena porcentagem, com base nos testes 
estatísticos, os autores sugerem que há risco aumentado de CIUR para mulheres que gestam em idade avançada, com destaque para as de 40 anos ou mais.

Em relação à hipertensão arterial pré-existente, neste estudo, encontrou-se diferença significativa com maiores médias de idade materna. Acredita-se que, ao envelhecer, haja uma perda gradual da complacência do sistema cardiovascular, devido a uma diminuição da capacidade de resposta vasodilatadora dependente do endotélio, que provoca maior resistência vascular periférica total, podendo levar ao aumento da pressão. Por isso, a predisposição das mulheres em idade avançada a complicações hipertensivas na gravidez pode ser explicada pela dificuldade do sistema cardiovascular de se adaptar à condição da mulher nessa situação, o que causa alterações hemodinâmicas que comprometem as trocas gasosas e de nutrientes ao feto ${ }^{(15)}$.

Dessa forma, as síndromes hipertensivas, assim como a DMG são igualmente associadas a desfechos negativos em relação ao recém-nascido. Os mais frequentes são prematuridade, baixo peso ao nascer ${ }^{(2-3,8-9)}$, internação em Unidade de Terapia Intensiva Neonatal ${ }^{(2,8-9)}$. Apesar de estes últimos aspectos não terem feito parte da análise deste estudo, considera-se imprescindível que seja dada a devida importância para a avaliação desses riscos.

Embora sem significância estatística neste estudo, PP e hemorragias são importantes complicações que estão associadas à idade da mulher na gestação. Considera-se importante dar atenção a esses dois desfechos, especialmente em mulheres que apresentam distúrbios hipertensivos. As situações hemorrágicas podem acontecer tanto no primeiro quanto no segundo trimestre da gestação. Abortamento, gravidez ectópica, descolamento prematuro de placenta, rotura uterina e PP são algumas das principais causas de hemorragias durante a gestação ${ }^{(1)}$. Já as que ocorrem no pós-parto podem ser desencadeadas por atonia uterina, trauma cirúrgico relacionado à cesárea e, também, por PP ou placenta acreta. Além disso, a idade materna avançada, a multiparidade $^{(13)}$ e as síndromes hipertensivas, incluindo a PE, são fatores de risco importantes que contribuem para a ocorrência de hemorragias ${ }^{(11)}$.

Assim, fica claro, na literatura, que, embora as situações hemorrágicas sejam um agravo obstétrico multicausal e não estejam invariavelmente ligadas à PP, à idade materna avançada e aos distúrbios hipertensivos, em algum nível, todos esses problemas apresentam relação fisiológica. Todos esses aspectos mostram-se interligados e com maiores probabilidades de ocorrerem em mulheres com idade avançada.

Os problemas apresentados mostram que as complicações a que estão submetidas as mulheres perpassam o risco de morbidade materna, mas também têm consequências para a saúde do feto/bebê. Por isso, torna-se mais grave e necessária a atenção a essa população. Nesse sentido, tem-se o enfermeiro como uma peça-chave, para que se estabeleça uma dinâmica de cuidados tanto físicos e biológicos quanto de diálogo, acolhimento e $\operatorname{escuta}^{(7)}$.

Quanto às outras variáveis relacionadas às complicações analisadas, nenhuma mostrou relação significativa com as faixas etárias pesquisadas. Todavia, requerem cuidado e cautela para que sejam avaliadas de forma a considerar a individualidade de cada mulher. Tendo em vista todos os fatores que indicam o aumento inerente ao risco em gestações tardias, é necessária atenção redobrada, a fim de evitar futuros resultados indesejáveis tanto para a mãe quanto para o bebê.

Dentre os dados sociodemográficos, a única variável que apresentou diferença significativa, quando relacionada aos grupos etários, foi a escolaridade. Uma revisão integrativa da literatura $^{(5)}$ mostrou, entre outros fatores, que o perfil de mulheres que engravidam com 35 anos ou mais, em âmbito internacional, apresenta grau de escolaridade elevado, trabalho com boa remuneração, estabilidade conjugal e planejamento da gravidez. No entanto, este estudo demonstra que apenas 175 (14,6\%) das participantes apresentaram nível de escolaridade com mais de 12 anos de estudo, o que configura ingresso no Ensino Superior. Os outros aspectos citados na revisão não foram analisados aqui, mas estudos nacionais demonstram que o Brasil não apresenta $\mathrm{O}$ 
perfil de maior instrução, tampouco das demais características, como causas para o adiamento da maternidade ${ }^{(10,16)}$.

$\mathrm{O}$ aspecto escolaridade pode ser um fator de risco para o desenvolvimento de complicações maternas, incluindo principalmente $\mathrm{PE}$ e $\mathrm{DMG}^{(8-9)}$. Além disso, é uma condição que, associada ao padrão socioeconômico, é responsável por interferir na compreensão sobre o próprio quadro de saúde ${ }^{(10)}$. No caso de mulheres em gestação tardia, embora muitas não apresentem sinais físicos de resultados das complicações, precisam ter clara a importância do acompanhamento regular e rigoroso do pré-natal, principalmente se a morbidade estiver relacionada à hipertensão arterial ou DMG, pois podem desencadear descompensação sistêmica com necessidade de intervenções mais invasivas.

Sob outra perspectiva, estudo ${ }^{(17)}$ encontrou efeito protetivo da escolaridade em mulheres gestantes com 41 anos ou mais. Apontou que as mulheres com maior grau de instrução tinham riscos semelhantes, ou até mesmo menores, de resultados adversos na gestação, quando comparadas àquelas com 21 a 34 anos. Os autores sugerem que esse efeito pode ter decorrido da associação indireta com os fatores econômicos, bem como da associação direta com a capacidade de compreensão da mulher sobre as orientações recebidas dos profissionais da saúde.

O padrão econômico, neste estudo, foi analisado com base na variável renda. Embora sem significância estatística, evidenciou o perfil monetário das mulheres atendidas no hospital pesquisado. Destaca-se que 415 prontuários tiveram esse dado preenchido, configurando pouco mais da metade da amostra. Desses, 222 (53,5\%) informaram que as mulheres recebiam de dois a três salários mínimos, o que, mesmo para os padrões brasileiros, é uma faixa de renda baixa, sendo classificada como classe D.

Salienta-se que o cenário de pesquisa é público e atende, em sua totalidade, por meio do SUS. Assim, entende-se que o nível socioeconômico e a escolaridade representam a população atendida nesse tipo de serviço. É importante frisar que $921(68,9 \%)$ prontuários tiveram o dado relacionado à renda negligenciado. É necessário atentar a essa lacuna, pois trata-se de um indicador de risco social que pode limitar o profissional na elaboração de um plano assistencial.

Esse resultado soma-se a outras características sociodemográficas de mulheres com gravidez tardia que vão de encontro ao perfil das gestantes tardias relatado por estudos internacionais ${ }^{(17-18)}$. Em contrapartida, os estudos nacionais estão de acordo com as características apresentadas nesta pesquisa, pois o Brasil, apesar de acompanhar a tendência da postergação da gestação, ainda é um país em desenvolvimento que destoa quanto a esses motivos de adiamento da gravidez $^{(5,8,10,16)}$.

Em relação às características obstétricas, apesar de nenhuma ter apresentado associação significativa, ainda assim, julga-se importante argumentar que os dados mostrados na literatura divergem dos aqui expostos. Especialmente quanto à via de parto, pondera-se que autores mostram o aumento da idade materna estritamente relacionado à prevalência de cesáreas, tanto eletivas quanto emergenciais ${ }^{(3-4,9,19)}$.

Neste estudo, não foram realizados testes mais acurados para averiguar essa relação. A frequência de cesáreas foi de 58,4\% e, apesar de sugerir que é um número relativamente alto, está próximo à taxa nacional de 55,6\%. Os dados do presente estudo condizem com a realidade brasileira, sugerindo que a amostra pesquisada pode ser representativa nesse aspecto. Nesse cenário, a Organização Mundial da Saúde (OMS) afirma que taxas de cesárea acima de 10\% não diminuem a mortalidade materna e neonatal, portanto, não trazem benefícios quando utilizadas de forma arbitrária. Apesar disso, também afirma que a preocupação dos órgãos de saúde deve ser relacionada à garantia da indicação correta $e$ necessária da cesárea, em detrimento da busca por uma taxa ideal específica ${ }^{(20)}$.

Várias são as condições pré-existentes da mulher que podem desencadear problemas na gestação. Por isso, a equipe de saúde e, especialmente, o enfermeiro devem estar atentos à coleta de dados durante as consultas de pré-natal, para identificar complicações reais e potenciais da mulher e, assim, realizar o planejamento do cuidado necessário a cada uma ${ }^{(7)}$. 
Como limitações, destacam-se a incompletude das informações nos prontuários, principalmente as relacionadas à renda, como também o fato de os dados terem sido preenchidos por outros profissionais, impossibilitando confirmar sua precisão. Além disso, o fato de não haver um grupo comparador que estivesse na faixa etária julgada ideal para a gestação (20 a 34 anos) e a não avaliação da relação entre as complicações maternas e os desfechos neonatais também podem ser considerados limitantes, para que outras análises pudessem ser realizadas. Ainda, pode ser considerada uma limitação, a coleta de dados em uma única instituição, o que dificulta a generalização dos achados. Contudo, por se tratar de um hospital de grande porte, com serviço de referência para o estado do Paraná, anseia-se que os resultados também sejam condizentes com outros cenários e possam contribuir para a melhoria dos cuidados com as gestantes em idade avançada.

\section{Conclusão}

As gestantes de 40 anos ou mais apresentavam maior probabilidade de desenvolvimento de CIUR e PE. Com o aumento da idade, é possível que HAS, PE e DMG ocorram com maior frequência. Diante disso, fica clara a necessidade de que os profissionais da saúde, notadamente a enfermagem, somem esforços para prevenir e intervir em desfechos potencialmente desfavoráveis e capazes de resultar em óbitos fetais e/ou maternos, bem como formular e seguir protocolos assistenciais e políticas públicas, a fim de se instrumentalizarem e promoverem melhorias na qualidade do cuidado dessas gestantes. Acredita-se que a gestação em idade avançada, por ser um fenômeno que cresce cada vez mais no mundo, embora com perfis diferentes em cada região, deva ser alvo de estudos pela enfermagem, pois tende a ser uma realidade recorrente em breve.

\section{Colaborações:}

1 - concepção, projeto, análise e interpretação dos dados: Juliane Dias Aldrighi, Suelen da Silva Ribeiro, Andressa Kachel Chemim,
Marilene Loewen Wall e Samuel Spiegelberg Zuge;

2 - redação do artigo e revisão crítica relevante do conteúdo intelectual: Juliane Dias Aldrighi, Marilene Loewen Wall e Adriana Aparecida Piler;

3 - aprovação final da versão a ser publicada: Juliane Dias Aldrighi, Suelen da Silva Ribeiro, Andressa Kachel Chemim, Marilene Loewen Wall, Samuel Spiegelberg Zuge e Adriana Aparecida Piler.

\section{Referências}

1. Brasil. Ministério da Saúde. Secretaria de Atenção à Saúde. Departamento de Ações Programáticas Estratégicas. Gestação de alto risco: manual técnico [Internet]. 5a ed. Brasília (DF); 2012 [cited 2021 Jan 10]. Available from: https://bvsms.saude. gov.br/bvs/publicacoes/gestacao_alto_risco.pdf

2. Lean SC, Derricott H, Jones RL, Heazell AEP. Advanced maternal age and adverse pregnancy outcomes: A systematic review and meta-analysis. PloS One. 2017;12(10):e0186287. DOI: https://doi. org/10.1371/journal.pone.0186287

3. Laopaiboon $M$, Lumbiganon $P$, Intarut $N$, Mori R, Ganchimeg T, Vogel JP, et al. Advanced maternal age and pregnancy outcomes: a multicountry assessment. BJOG. 2014;121(Suppl 1):49-56. DOI: https://doi.org/10.1111/1471-0528.12659

4. Haslinger C, Stoiber B, Capanna F, Schäffer MK, Zimmermann R, Schäffer L. Postponed pregnancies and risks of very advanced maternal age. Swiss Med Wkly. 2016;146:w14330. DOI: https://doi.org/10.4414/smw.2016.14330

5. Aldrighi JD, Wall ML, Souza SRRK, Cancela FZV. The experiences of pregnant women at an advanced maternal age: an integrative review. Rev Esc Enferm USP. 2016;50(3):512-21. DOI: https://doi.org/10.1590/ S0080-623420160000400019

6. Brasil. Ministério da Saúde. Departamento de Informática do Sistema Único de Saúde (DATASUS). Número de nascidos vivos conforme a idade materna 2007-2017 [Internet]. Brasília (DF): 2021 [cited 2021 Jan 10]. Available from: http:// tabnet.datasus.gov.br/cgi/deftohtm.exe?sinasc/ cnv/nvuf.def

7. Souza BF, Bussadori JCC, Ayres JRCM, Fabbro MRC, Wernet M. Nursing and hospitalized 
high-risk pregnant women: challenges for comprehensive care. Rev Esc Enferm USP. 2020;54:e03557. DOI: https://doi.org/10.1590/ S1980-220X2018036903557

8. Alves NCC, Feitosa KMA, Mendes MES, Caminha MFC. Complications in pregnancy in women aged 35 or older. Rev Gaúcha Enferm. 2017;38(4):e2017-0042. DOI: http://dx.doi.org/ 10.1590/1983-1447.2017.04.2017-0042

9. Shan D, Qiu PY, Wu YX, Chen Q, Li AL, Ramadoss S, et al. Pregnancy outcomes in women of advanced maternal age: a retrospective cohort study from China. Sci Rep. 2018;8(1):12239. DOI: https://doi.org/10.1038/s41598-018-29889-3

10. Aldrighi JD, Ribeiro SS, Wall ML, Zuge SS, Souza SRRK, Piler AA. Perfil sociodemográfico e obstétrico de mulheres em idade materna avançada. Rev enferm UFSM. 2018;8(3):423-37. DOI: https://doi.org/10.5902/2179769225922

11. Ruiz MT, Azevedo CT, Ferreira MBG, Mamede MV. Association between hypertensive disorders and postpartum hemorrhage. Rev Gaúcha Enferm. 2015;36(n esp):55-61. DOI: https://doi.org/10.1590/1983-1447.2015.esp.56776

12. Martinelli KG, Garcia ÉM, Santos Neto ETD, Gama SGND. Advanced maternal age and its association with placenta praevia and placental abruption: a meta-analysis. Cad Saúde Pública. 2018;34(2):e00206116. DOI: https://doi. org/10.1590/0102-311X00206116

13. Huque S, Roberts I, Fawole B, Chaudhri R, Arulkumaran S, Shakur-Still1 H. Risk factors for peripartum hysterectomy among women with postpartum haemorrhage: analysis of data from the WOMAN trial. BMC Pregnancy Childbirth. 2018;18:186. DOI: https://doi.org/10.1186/ s12884-018-1829-7

14. American College of Obstetricians and Gynecologists. Practice Bulletin n. 204. Fetal growth restriction. Obstetrics \& Gynecology. 2019;133(2):e97-e109. DOI: https://doi.org/10. 1097/AOG.0000000000003071

15. Roustaeia Z, Vehviläinen-Julkunenb K, Tuomainena TP, Lamminpääc R, Heinonen S. The effect of advanced maternal age on maternal and neonatal outcomes of placenta previa: A register-based cohort study. Eur J Obstet Gynecol Reprod Biol. 2018;227:1-7. DOI: https://doi.org/10.1016/j.ejogrb.2018.05.025

16. Aldrighi JD, Wall ML, Souza SRRK. Experience of pregnant women at an advanced age. Rev Gaúcha Enferm. 2018;39:e20170112. DOI: https://doi.org/10.1590/1983-1447.2018. 2017-0112

17. Almeida NKO, Almeida RMVR, Pedreira CE. Adverse perinatal outcomes for advanced maternal age: a cross-sectional study of Brazilian births. J Pediatr. 2015;91(5):493-8. DOI: https://doi. org/10.1016/j.jped.2014.12.002

18. Southby C, Cooke A, Lavender T. "It's now or never" - nulliparous women's experiences of pregnancy at advanced maternal age: a grounded theory study. Midwifery. 2019;68:1-8. DOI: https:// doi.org/10.1016/j.midw.2018.09.006

19. Rydahl E, Declercq E, Juhl M, Maimburg RD. Cesarean section on a rise-Does advanced maternal age explain the increase? A population register-based study. PloS One. 2019;14(1):e0210655. DOI: https://doi.org/10.1371/ journal.pone.0210655

20. World Health Organization. Human Reproduction Programme. WHO Statement on Caesarean Section Rates [Internet]. Geneva; 2015 [cited 2021 Jan 10]. Available from: https:// apps.who.int/iris/bitstream/handle/10665/ 161442/WHO_RHR_15.02_eng.pdf;jsessionid= EA464EF4BD41EDD2858814435E51508D? sequence $=1$

Recebido: 15 de janeiro de 2021

Aprovado: 26 de abril de 2021

Publicado: 13 de maio de 2021

A Revista Baiana de Enfermagem utiliza a Licença Creative Commons - Atribuição-NãoComercial 4.0 Internacional. https://creativecommons.org/licenses/by-nc/4.0/ Este artigo é de acesso aberto distribuído sob os termos da Licença Creative Commons (CC BY-NC). Esta licença permite que outros remixem, adaptem e criem a partir do seu trabalho para fins não comerciais. Embora os novos trabalhos tenham de lhe atribuir o devido crédito e não possam ser usados para fins comerciais, os usuários não têm de licenciar esses trabalhos derivados sob os mesmos termos. 